\title{
Effects of Short-Term Abstinence from Escalating Doses of D-Amphetamine on Drug and Sucrose-Evoked Dopamine Efflux in the Rat Nucleus Accumbens
}

\author{
Giada Vacca', Soyon Ahn' and Anthony G Phillips*,' \\ 'Department of Psychiatry, Institute of Mental Health, University of British Columbia, Vancouver, BC, Canada
}

\begin{abstract}
Abstinence from high doses of psychostimulant drugs, in both humans and rodents, is linked to adverse psychological effects including anhedonia, a core symptom of major depression, manifested behaviorally as decreased responding for rewarding stimuli. The present study used brain microdialysis in freely moving rats to examine the effect of D-amphetamine (D-amph) withdrawal on changes in extracellular dopamine (DA) levels in the nucleus accumbens (NAc) evoked by D-amph or behavior related to sucrose consumption. D-amph was administered intraperitoneally (i.p.) according to an escalating dose (ED) schedule (from I to $10 \mathrm{mg} / \mathrm{kg}, 3 \mathrm{doses} / \mathrm{day}$ ). We first confirmed the development of tolerance by monitoring DA efflux in the NAc in response to 5 and $10 \mathrm{mg} / \mathrm{kg}$ doses of D-amph administered during the ED schedule of drug administration and again in response to the $5 \mathrm{mg} / \mathrm{kg}$ dose of D-amph $72 \mathrm{~h}$ following the last $10 \mathrm{mg} / \mathrm{kg}$ D-amph injection. In a separate study, DA efflux in the NAc was first shown to be increased significantly during both preparatory and consummatory phases of responding for a $4 \%$ sucrose solution. Withdrawal from the ED schedule of D-amph caused a selective attenuation of DA efflux only during the preparatory phase of the sucrose test. These results provided convincing evidence of neurochemical adaptation within the mesocorticolimbic DA pathway during and following the administration of an ED schedule of D-amph as well as suppressed neurochemical responses to a psychostimulant drug and cues associated with a natural reward after withdrawal from drug treatment. Accordingly, these findings support the hypothesis that downregulation of mesocorticolimbic DA function maintained during D-amph withdrawal may account for the selective disruption of motivated behavior reported in studies employing psychostimulant drug withdrawal as a model of depression in rodents.

Neuropsychopharmacology (2007) 32, 932-939. doi:I0.1038/sj.npp. I 301 I73; published online 2 August 2006
\end{abstract}

Keywords: microdialysis; dopamine; motivated behavior; escalating doses of D-amphetamine; tolerance; psychostimulant withdrawal

\section{INTRODUCTION}

Several studies have shown that short-term abstinence from sustained exposure to high doses of psychostimulant drugs such as cocaine or D-amphetamine (D-amph) may precipitate psychological (Gawin and Kleber, 1986; Weddington et al, 1990; Kampman et al, 1998) and somatic (Srisurapanont et al, 1999) effects that share many of the features of major depressive disorder (MDD) in humans. Preclinical studies of the effects of cessation of repeated administration of psychostimulants in rodents have demonstrated many behavioral changes that conform to key symptoms of MDD in humans, including the core symptom

*Correspondence: Dr AG Phillips, Department of Psychiatry, Institute of Mental Health, University of British Columbia, 2255 Wesbrook Mall, Vancouver, BC, Canada V6T 2AI, Tel: + I 604822 4624, Fax: + I 604 822 7756, E-mail: aphillips@psych.ubc.ca

Received 24 October 2005; revised 14 June 2006; accepted 20 June 2006

Online publication: 28 June 2006 at http://www.acnp.org/citations/ Npp062806050644/default.pdf of anhedonia (see Barr et al, 2002, for a review). Anhedonia may be defined as 'a markedly diminished interest or pleasure in all or most activities' (Gardner, 2000) and has been modeled in experimental animals as a failure to maintain responding for natural or artificial reward stimuli (Wise, 1978).

Rats recently withdrawn from chronic treatment with psychostimulant drugs display decreased responding for rewarding electrical brain stimulation reward, which is attributed to diminished activity in the neural substrates of reward (Kokkinidis et al, 1980; Markou and Koob, 1991; Lin et al, 1999). Recent findings from our laboratory have shown that during withdrawal from an escalating dose (ED) schedule of D-amph administration $(1-10 \mathrm{mg} / \mathrm{kg})$, rats also exhibit reduced motivation to obtain natural reinforcers, including a sucrose solution (Barr and Phillips, 1999) or a sexually receptive conspecific (Barr et al, 1999), for up to 5 days following the termination of drug treatment. Furthermore, rats withdrawn from the same regimen of $\mathrm{D}$-amph injections showed increased successive negative contrast (Barr and Phillips, 2002), and failed to display successive 
positive contrast (Vacca and Phillips, 2005). These results provide compelling evidence that, in addition to deceased motivation to respond for artificial and natural reward stimuli, animals withdrawn from an ED schedule of psychostimulant drug treatment also have a generalized incapacity to respond to unexpected gains or reductions in reward value.

A major brain reward pathway arises from dopaminergic neurons in the ventral tegmental area of the midbrain and projects mainly to the nucleus accumbens (NAc) and medial prefrontal cortex (Mogenson and Phillips, 1978; Phillips and Fibiger, 1978). This mesocorticolimbic dopamine (DA) pathway plays a critical role in many aspects of reward, including motivational states triggered by natural incentive stimuli (eg, food, sex, and social interaction) or pharmacological stimuli that can maintain drug self-administration (Everitt et al, 1999; Koob et al, 1998; Wise, 1998). In vivo microdialysis studies in rats report increased DA efflux in the NAc during preparatory and consummatory phases of feeding and sexual behaviors (Phillips et al, 1993; Wilson et al, 1995; Fiorino et al, 1997; Ahn and Phillips, 1999). Furthermore, virtually all drugs of abuse increase DA transmission in the NAc, which partly mediates their rewarding effects (Koob et al, 1998; Wise, 1998). Therefore, it is possible that some of the depressive-like symptoms observed during psychostimulant withdrawal in rodents, including anhedonia and decreased motivation, may result from reduced activity in the mesocorticolimbic DA system in the form of decreased efflux of DA in the NAc.

The hypothesis that DA efflux in the NAc, evoked by pharmacological stimuli and natural rewards, is attenuated following withdrawal from an ED schedule of D-amph was tested using in vivo microdialysis coupled with highperformance liquid chromatography with electrochemical detection (HPLC-EC). In the first experiment, rats were challenged with a $5-\mathrm{mg} / \mathrm{kg}$ injection of D-amph 72 -h after the ED treatment. The development of tolerance to the indirect DA agonist property of D-amph ( 5 and $10 \mathrm{mg} / \mathrm{kg}$ ) was also monitored during the ED schedule of D-amph injections. A second experiment examined the effect of withdrawal from D-amph on DA efflux in the NAc associated with sucrose reward, during preparatory, consummatory, and post-consummatory phases of responding for this natural reward.

\section{MATERIALS AND METHODS}

\section{Subjects}

Male Long-Evans rats (Charles River, Quebec, Canada), weighing 250-300 g, were pair-housed for a minimum of 1 week before surgery and then housed individually following surgery in a temperature-controlled colony $\left(21^{\circ} \mathrm{C}\right)$ with reverse light/dark cycle conditions (lights on 0400-1600). Food and water were available ad libitum, unless otherwise indicated. All training and testing sessions were conducted during the dark cycle. All experimental procedures were performed in accordance with the standards of the Canadian Council on Animal Care and approved by the Committee on Animal Care, University of British Columbia.

\section{Surgery}

All rats were anesthetized with intraperitoneal (i.p.) injections of ketamine hydrochloride $(100 \mathrm{mg} / \mathrm{kg})$ and xylazine $(7 \mathrm{mg} / \mathrm{kg})$ and implanted bilaterally with stainless-steel guide cannulae (19 G, $15 \mathrm{~mm}$ length) positioned $1 \mathrm{~mm}$ below dura, directly over the NAc $(+1.7 \mathrm{~mm} \mathrm{AP}$ and $\pm 1.1 \mathrm{~mm}$ ML from bregma). Coordinates were determined according to Paxinos and Watson brain atlas (1997). Animals were allowed to recover for 1 week before being assigned to Experiment 1 or Experiment 2.

\section{ED Protocol of D-Amph Administration}

Different doses of D-amph sulfate (Sigma, St Louis, MO) were prepared daily by dissolving the appropriate amount of drug in $1 \mathrm{ml}$ isotonic saline $(0.9 \% \mathrm{NaCl})$. The injection volume for each rat was adjusted on a daily basis according to the rat's weight. In all experiments, D-amph was administered (i.p.) using an ED protocol shown to minimize the chance of acute toxicity associated with high doses of D-amph (Ryan et al, 1990). Rats received injections at 0800, 1500 , and 2200 for 5 consecutive days. On Day 1, rats received an initial dose of $1 \mathrm{mg} / \mathrm{kg}$ and on Days 2-4, nine subsequent doses that each increased by $1 \mathrm{mg} / \mathrm{kg}$ (last dose $10 \mathrm{mg} / \mathrm{kg}$ ). On Day 5, rats received two additional doses of $10 \mathrm{mg} / \mathrm{kg}$.

\section{Microdialysis Procedure and HPLC}

Microdialysis probes were concentric in design with silica inlet-outlet lines. The active surface consisted of a semipermeable membrane $2 \mathrm{~mm}$ in length $(340 \mu \mathrm{m}$ outer diameter; 65000 molecular weight cutoff; Filtral 12; Hospal, Neurnberg, Germany). Typical in vitro probe recoveries of DA conducted at room temperature were $18 \pm 1 \%$ of a standard DA solution. Before implantation, probes were connected to an Instech dual-channel liquid swivel (Plymouth Meeting, PA) and continuously perfused at $1 \mu \mathrm{l} / \mathrm{min}$ with artificial cerebrospinal fluid (aCSF). The aCSF consisted of a $10.0 \mathrm{mM}$ sodium phosphate buffer with $147.0 \mathrm{mM} \mathrm{NaCl}, 3.0 \mathrm{mM} \mathrm{KCl}, 1.0 \mathrm{mM} \mathrm{MgCl}_{2}$, and $1.2 \mathrm{mM}$ $\mathrm{CaCl}_{2}$ (pH 7.4). Probes were implanted in the NAc via the guide cannulae, with the dialyzing membrane extending from -5.8 to $-7.8 \mathrm{~mm}$ DV from dura, and perfused overnight $(\sim 14-16 \mathrm{~h})$ at $1 \mu \mathrm{l} / \mathrm{min}$ with aCSF.

DA content in NAc dialysates was analyzed using HPLCEC. The HPLC system consisted of an ESA 582 pump (ESA Inc., Bedford, MA), a pulse damper (Scientific Systems Inc., State College, PA), a Rheodyne Inert manual injector (model 9125i, $20 \mu$ l injection loop; Rohnert Park, CA), a Tosoh Bioscience Super ODS TSK column $(2 \mu \mathrm{m}$ particle, $2 \times 10 \mathrm{~mm}$; Montgomeryville, PA) and an Antec Leyden Intro Electrochemical detector and VT-03 flow cell with a $\mathrm{Ag} / \mathrm{AgCl}$ reference electrode $\left(V_{\text {applied }}=+700 \mathrm{mV}\right.$; Leiden, The Netherlands). The mobile phase $(70 \mathrm{mM}$ sodium acetate, $40 \mathrm{mg} / \mathrm{l} \mathrm{EDTA}$ and $50 \mathrm{mg} / \mathrm{l}$ of sodium octyl sulfate (adjustable), $\mathrm{pH} 4.0,12 \%$ methanol) flowed through the system at $0.18 \mathrm{ml} / \mathrm{min}$. EZChrome Elite software (Scientific Software, Pleasanton, CA) was used to acquire and analyze chromatographic data. 
Experiment 1: Changes in DA Efflux during and Following an ED Schedule of D-Amph Injections

One week following the implantation of bilateral NAc guide cannulae, one group of rats received ED of D-amph (EDtreated group) as described above (see Table 1). A separate group of rats was treated with isotonic saline (saline-treated group) under the same schedule as rats in the ED-treated group. The ED-treated group was tested with microdialysis in two of the following $\mathrm{D}$-amph injection conditions: during (i) the fifth injection $(5 \mathrm{mg} / \mathrm{kg})$, (ii) the 12 th injection $(10 \mathrm{mg} / \mathrm{kg})$, and (iii) the challenge injection $(5 \mathrm{mg} / \mathrm{kg}) 72 \mathrm{~h}$ following the completion of the ED schedule of D-amph treatment. The saline-treated group was tested in two of the following saline injection conditions: (i) the first injection (acute saline group), (ii) the fifth injection, (iii) the 12th injection, and (iv) challenge injection $72 \mathrm{~h}$ following the final scheduled saline injection. Each rat was implanted with a probe in one NAc (either the right or left hemisphere) for the first microdialysis test, and then the contralateral NAc for the second test.

Two separate groups of rats, without prior treatment history, received a single injection of either 5 or $10 \mathrm{mg} / \mathrm{kg}$ D-amph (i.p.) during a microdialysis experiment (acute groups). Acute groups received only one microdialysis session.

On each test day, samples were collected at 30-min intervals and analyzed immediately for DA using HPLC. When three consecutive baseline samples showed less than $10 \%$ fluctuation, rats were administered the appropriate injection (D-amph or saline) and dialysates were collected for a further $240 \mathrm{~min}$.

\section{Experiment 2: Effect of D-Amph Withdrawal on} Sucrose-Evoked DA Efflux

Rats in Experiment 2 were divided into two groups: the ED-treated group received ED of D-amph as described in Table 2. The control group received matched saline injections in accordance with the timing of the ED schedule (saline-treated group). All rats were trained to drink a $4 \%$ sucrose solution during a single 20 -min period in the testing chamber. A removable Plexiglas screen perforated with holes $(1 \mathrm{~cm}$ diameter $)$ divided each chamber into a smaller $(16 \times 38 \times 38 \mathrm{~cm})$ and a larger compartment $(25 \times 38 \times 38 \mathrm{~cm})$. Rats were food deprived for $12 \mathrm{~h}$ before each training session. All the training sessions occurred between 0900 and 1000. Each day, 4\% (w/v) sucrose solutions were freshly prepared and made available via a drinking spout protruding into the smaller compartment of the cage. Each training session consisted of two phases. During the preparatory phase, rats were confined to the larger compartment by the screen for $10 \mathrm{~min}$ and could not make contact with the sucrose. The consummatory phase began with the removal of the screen, giving access to the sucrose solution for $20 \mathrm{~min}$. Removal of the drinking spout marked the beginning of a 30-min post-consummatory phase.

Once latencies to initiate sucrose intake following removal of the screen had stabilized (in most cases by Day 3), the next day, a sucrose drinking test was conducted in conjunction with concurrent microdialysis sampling from the NAc (see Table 2). Samples were collected every $5 \mathrm{~min}$ and immediately analyzed for DA by HPLC. When three consecutive baseline samples showed less than 5\% fluctuation, the spout containing sucrose was inserted through the back wall of the smaller compartment, initiating the preparatory phase. The perforated screen was removed after $10 \mathrm{~min}$ and dialysates were collected for $20 \mathrm{~min}$ with sucrose available and for an additional $30 \mathrm{~min}$ following the end of the consummatory phase. On Days 5-9, one group of rats was administered D-amph, and one group saline, according to the ED protocol. On Day $12,72 \mathrm{~h}$ after the last D-amph or saline injection, rats were tested with microdialysis during the preparatory, consummatory, and post-consummatory phases of sucrose consumption.

Table I Experiment | Protocol

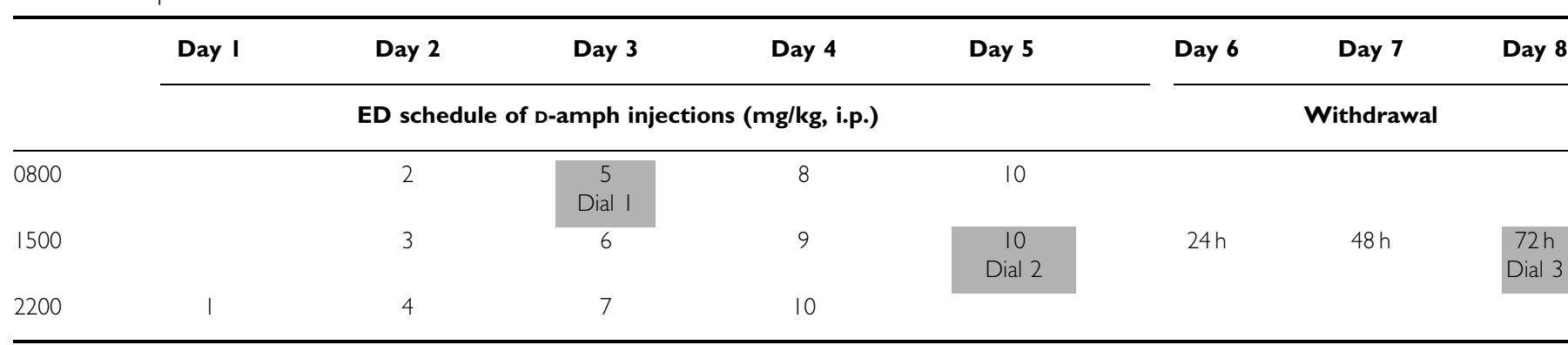

Table 2 Experiment 2 Protocol

\begin{tabular}{|c|c|c|c|c|c|c|c|c|c|c|c|c|}
\hline & Day I & Day 2 & Day 3 & Day 4 & \multicolumn{5}{|c|}{ ED schedule of D-amph injections (mg/kg, i.p.) } & \multicolumn{3}{|c|}{ Withdrawal } \\
\hline 1500 & & & & & I & 4 & 7 & 10 & & & & \\
\hline
\end{tabular}




\section{Histology}

Following the last microdialysis experiment, rats were deeply anesthetized with sodium pentobarbital (100 mg/ $\mathrm{ml}$ ) and perfused intracardially with $0.9 \% \mathrm{NaCl}$. Brains were removed immediately and stored in $20 \%$ (w/v) sucrose and $4 \%$ paraformaldehyde solution for 1 week, sliced into $50 \mu \mathrm{m}$ coronal sections, and then stained with cresyl violet. Placements of probes were verified according to the atlas of Paxinos and Watson (1997). Only animals with tracts in the shell/core region of the NAc were included in the statistical analysis.

\section{Data Analyses}

The mean concentrations of DA in the final three baseline samples before treatment were normalized $(0 \%)$ and all data were expressed as \% change from this baseline value. Statistical significance of the neurochemical data was evaluated using a one- or two-way repeated measures analysis of variance (ANOVA) with Time as the within- and Group as the between-subjects factor. This was followed, when appropriate, by the Dunn (for between-groups comparisons) or Dunnett (within-group comparisons) method of multiple comparisons. In the latter test, the baseline sample preceding experimental manipulation was used as the control value. The Huynh-Feldt correction for nonsphericity was applied to the degrees of freedom for all within-subject analyses. Analyses were performed using Systat statistical software.

\section{RESULTS}

\section{Experiment 1: Changes in DA Efflux during and Following an ED Schedule of D-Amph Injections}

As shown in Figure 1a, injection of a $5 \mathrm{mg} / \mathrm{kg}$ (i.p.) dose of $\mathrm{D}$-amph resulted in a significant change in DA efflux in the NAc of rats that received $D$-amph for the first time (acute group, $n=5$ ), as well as those that received the dose as the fifth injection of the ED schedule (ED-treated group, $n=7$ ), but DA efflux was altered neither by an acute saline injection $(n=4)$ nor by the fifth injection of saline in salinetreated rats $(n=3)\left(\mathrm{F}_{(27,135)}=32.498, p<0.001\right)$. The peak magnitude of D-amph-evoked DA efflux $(+972 \%$ above baseline, observed 60-min post-injection) in the ED-treated group was significantly smaller than the maximum effect observed in the acutely treated rats $(+3324 \%$ above baseline; Dunn's $p<0.05)$. The D-amph-evoked increases remained significantly elevated above baseline for $\sim 3 \mathrm{~h}$ in both groups (Dunnett's, $p<0.05$ ).

During a separate series of microdialysis sessions similar group differences were observed in the maximum increase in DA efflux evoked by a $10 \mathrm{mg} / \mathrm{kg}$ (i.p.) dose of D-amph, as either the final dose of the ED schedule $(n=6)$ or as an acute dose of D-amph $(n=5)$, but DA efflux was not altered by an acute injection of saline $(n=4)$, or by the 12 th saline injection in saline-treated rats $(n=3)\left(\mathrm{F}_{(27,126)}=31.436\right.$, $p<0.001$; Figure 1b). These significant increases in DA efflux in the NAc remained elevated for over $3 \mathrm{~h}$ (Dunnett's, $p<0.05)$, and the peak increase observed 60-min postinjection in the ED-treated group ( $+1004 \%$ above baseline)
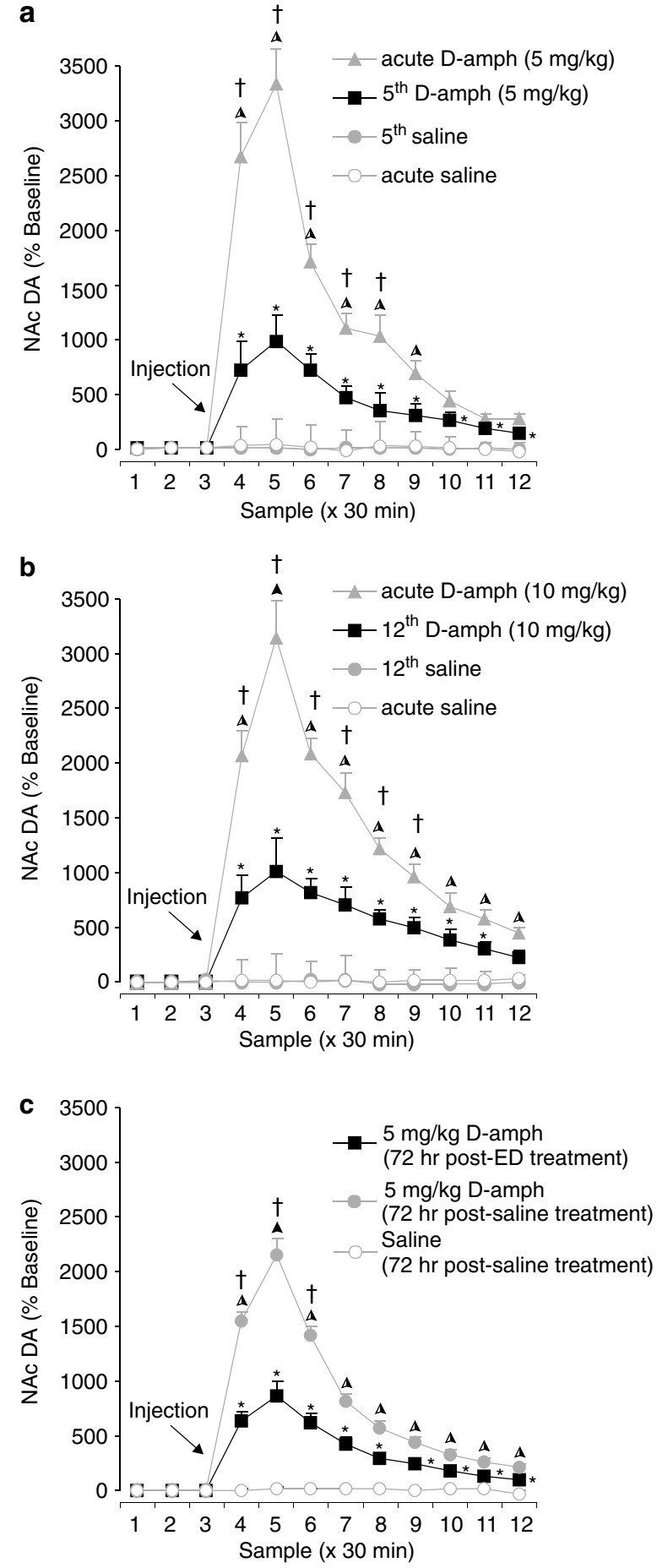

Figure I NAc DA efflux evoked by D-amph during or following an ED schedule of D-amph treatment. DA efflux was sampled by microdialysis and measured by HPLC-EC (mean + SEM) from rats receiving D-amph according to the ED schedule of drug treatment (black squares), ED schedule-matched saline injections (gray circles), acute D-amph injections of 5 or $10 \mathrm{mg} / \mathrm{kg}$ (gray triangles), and acute saline injections (white circles). DA efflux was monitored during the (a) fifth scheduled or acute injection of D-amph ( $5 \mathrm{mg} / \mathrm{kg}$, i.p.), (b) 12 th scheduled or acute injection ( $10 \mathrm{mg} / \mathrm{kg}$, i.p.), or (c) a $5 \mathrm{mg} / \mathrm{kg}$ (i.p.) challenge injection or saline injection $72 \mathrm{~h}$ following the final ED scheduled or saline treatment. Significant difference from

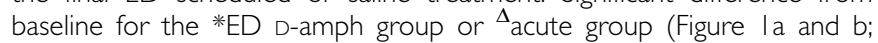
Dunnett's, $p<0.05)$. Significant difference from baseline *saline-treated group (Figure Ic; Dunnett's, $p<0.05$ ). †Significant difference between groups (Dunn's, $p<0.05$ ). 
was again significantly blunted compared to a $+3132 \%$ increase in the acute group given a single injection of $10 \mathrm{mg} / \mathrm{kg}$ D-amph (Dunn's $p<0.05$ ).

The final series of microdialysis sessions monitored differences in basal and D-amph- $(5 \mathrm{mg} / \mathrm{kg})$-evoked DA efflux in the NAc, $72 \mathrm{~h}$ following the last injection of D-amph or saline of the ED schedule (Figure 1c). In salinetreated rats, the average basal concentration of DA was $1.9 \mathrm{nM} \pm 0.21 / 9 \mu \mathrm{l}$ sample (uncorrected for probe recovery). This parameter was not significantly altered by prior administration of the ED schedule to the ED-treated group $(2.5 \mathrm{nM} \pm 0.23$ per $9 \mu \mathrm{l}$ sample). Injection of a $5 \mathrm{mg} / \mathrm{kg}$ (i.p.) dose of D-amph resulted in a significant change in DA efflux in both ED- $(n=7)$ and saline-treated $(n=9)$ groups $\left(\mathrm{F}_{(18,189)}=179.727, p<0.001\right)$. D-amph-induced DA efflux increased significantly above baseline in both groups (Dunnett's, $p<0.05$ ); however, the maximum change in DA efflux was significantly attenuated in the ED-treated group $(+838 \%)$ compared to the saline-treated group $(+2100 \%$; Dunn's, $p<0.05)$. Administration of a saline injection to saline-treated rats $(n=8)$ failed to induce any changes in DA efflux in the NAc.

\section{Experiment 2: Effect of Withdrawal from an ED D-Amph Schedule on Sucrose-Evoked DA Efflux}

The average basal concentration of DA in the presaline and preamph groups was $1.82 \mathrm{nM} \pm 0.30 / 5 \mu \mathrm{l}$ sample (uncorrected for probe recovery). This parameter was not significantly altered by the administration of either the ED schedule to the ED-treated group $(2.1 \pm 0.26 \mathrm{nM} / 5 \mu \mathrm{l} \mathrm{sam}$ ple) or saline to the saline-treated group $(1.97 \pm 0.20 \mathrm{nM} / 5 \mu \mathrm{l}$ sample).

As shown in Figure 2a, during the initial microdialysis sessions conducted before being treated on the ED schedule of D-amph injections $(n=6)$, or saline injections $(n=5)$, significant changes in DA efflux in the NAc were observed during the preparatory and consummatory phases of the sucrose test $\left(\mathrm{F}_{(12,108)}=4.657, p<0.001\right)$. In rats assigned to receive either $\mathrm{ED}$ or saline treatment (preamph or presaline groups, respectively), significant increase in DA efflux in the NAc associated with the preparatory phases was only observed in the first $5 \mathrm{~min}$ sample, whereas the enhanced DA efflux during sucrose consumption remained elevated for $15 \mathrm{~min}$ in both groups (Dunnett's, $p<0.05$ ). The latency to approach the drinking spout was $11.05 \pm 2.04 \mathrm{~s}$, whereas the volume of sucrose solution consumed during the 20-min test was $7 \pm 1.16 \mathrm{ml}$.

Seventy-two hours following withdrawal from treatment with the ED schedule of D-amph $(n=7)$ or saline $(n=5)$, significant changes in DA efflux were observed during both the preparatory and consummatory phases of the sucrose test (see Figure $2 \mathrm{~b})\left(\mathrm{F}_{(12,132)}=4.059, p<0.001\right)$. As noted in Figure $2 \mathrm{~b}$, significant increase in DA efflux from baseline was observed during the preparatory and consummatory phases of sucrose intake (Dunnett's, $p<0.05$ ) in both the ED- and saline-treated rats. The latency to approach the drinking spout was $17.23 \pm 2.60 \mathrm{~s}$, whereas the volume of sucrose solution consumed during the 20 -min test was $6.25 \pm 1.52 \mathrm{ml}$.

A more detailed comparison of the magnitude of change in DA efflux during the preparatory and consummatory
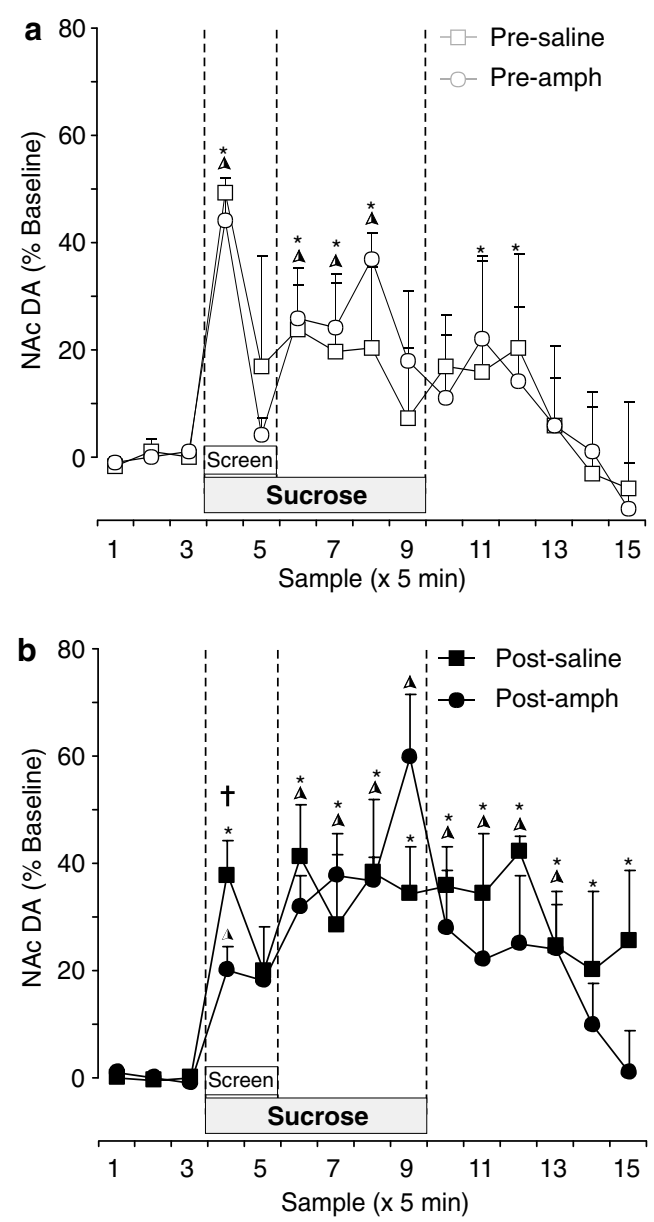

Figure 2 Changes in NAc DA efflux during a two-stage access to a 4\% sucrose solution, (a) before (white circles and white squares) and (b) $72 \mathrm{~h}$ following the final ED-scheduled injection of D-amph ( $1-10 \mathrm{mg} / \mathrm{kg}$, i.p.) (black circles) or the final saline injection (black squares). Dashed lines highlight the initial preparatory phase in which sucrose was presented behind a perforated screen (Samples 4 and 5) and the consummatory phase, when rats were given access to the sucrose following removal of the screen (Samples 6-9). Significant difference from baseline for ${ }^{\Delta}$ ED- rats and *saline-treated rats (Dunnett's, $p<0.05$ ). `Significant difference between ED- and saline-treated groups (Dunn's, $p<0.05$ ).

phases of the sucrose test in the ED- and saline-treated rats were conducted with separate two-way repeated measure ANOVAS. Results indicated a significant interaction of Group $\times$ Time only in the preparatory phase $\left(\mathrm{F}_{(3,20)}=3.697\right.$, $p<0.029)$. Post hoc analysis revealed that the increase in DA efflux during the first $5 \mathrm{~min}$ of the preparatory phase in the D-amph-treated rats was significantly lower than the increase in DA efflux observed in the saline-treated rats $(+38 v s+20 \%$; Dunn's, $p<0.05)$.

\section{Histology}

As illustrated in Figure 3, tracts of microdialysis probes were located close to the border of the shell/core regions of the NAc $(+1.6$ to $2.2 \mathrm{~mm} \mathrm{AP})$. In Experiment 1, neurochemical data from six NAc sites were excluded from statistical analyses owing to probe misplacement. In 


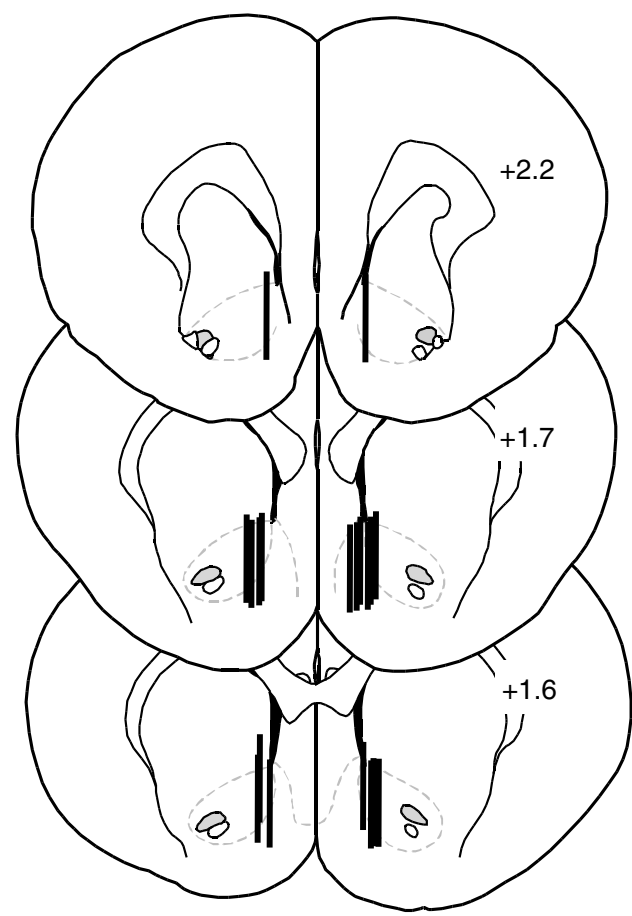

Figure 3 Placement of microdialysis probes in the NAc for those rats included in statistical analyses of neurochemical data. Vertical lines represent the $2 \mathrm{~mm}$ length of dialysis membrane. Distance from bregma is indicated (mm).

Experiment 2, data from three NAc site was excluded owing to failure of the rat to drink sucrose.

\section{DISCUSSION}

The present study provided neurochemical data that are complimentary to a growing body of behavioral studies showing marked reduction in responding for natural or artificial rewards shortly after withdrawal from an ED schedule of D-amph. Specifically, DA efflux in the NAc evoked by a pharmacological reward stimulus or cues presented during the preparatory but not consummatory phase of a sucrose test was attenuated in ED-treated rats, compared to saline-treated rats (Figure 1). This may reflect neurochemical adaptation within DA neurons of the mesocorticolimbic DA pathway, which in turn may underlie the development of tolerance to the indirect DA agonist property of D-amph. Interestingly, tolerance to the effect of D-amph on DA efflux developed rapidly, and was observed in response to fifth dose of the ED regimen $(5 \mathrm{mg} / \mathrm{kg}$, i.p.), and maintained in response to the final dose of D-amph $(10 \mathrm{mg} / \mathrm{kg}$, i.p.). Decreased extracellular levels of DA in the NAc have also been observed following withdrawal from extended treatment with cocaine (Weiss et al, 1992). In the present study, tolerance was maintained for $72 \mathrm{~h}$ following withdrawal from the drug, a time when symptoms of anhedonia and decreased motivation are observed in models of depression in rats using withdrawal from similar ED schedule of treatment with psychostimulants (Barr et al, 1999; Barr and Phillips, 1999, 2002). Using a chronic mild stress model of depression, Di Chiara et al (1999) similarly observed blunted mesolimbic DA efflux in response to appetitive rewarding stimuli. Collectively, these data are consistent with the hypothesis that synaptic transmission in the mesocorticolimbic DA system is significantly reduced following exposure to repeated treatment with high doses of psychostimulants, which in turn may account for the behavioral symptoms related to depression.

A direct comparison of the magnitude of DA efflux in the NAc evoked by a $5 \mathrm{mg} / \mathrm{kg}$ dose of $\mathrm{D}$-amph in saline-treated rats $72 \mathrm{~h}$ after the last saline injection (see Figure 1c) was approximately $\frac{1}{3}$ less than the magnitude of DA efflux evoked by the same dose administered to drug-naïve animals that had minimal handling and no prior injection history (see Figure 1a). These data imply a possible interaction between the effects of handling and/or injection stress and the pharmacological effects of D-amph on DA efflux in the NAc. Given the possible contribution of habituation to the stress of the injection protocol to the magnitude of DA efflux evoked by D-amph $72 \mathrm{~h}$ after withdrawal, it is important to note that the magnitude observed in ED D-amph condition $(+838 \%)$ was still significantly less than the value measured in control rats $(+2100 \%)$ with a similar treatment history.

A second experiment confirmed a significant increase in DA efflux in the NAc during both the preparatory and consummatory phases of sucrose intake by drug-naïve rats (Figure 2). In control tests conducted before treatment with either saline or an ED schedule of D-amph, the maximal increase in DA efflux occurred during the first 5 min of the preparatory phase preceding sucrose drinking (preamph group $+45 \%$, presaline group $+49 \%$ ). A similar pattern of DA efflux also was observed previously only in the first $5 \mathrm{~min}$ of the preparatory phase of feeding behavior (Wilson et al, 1995). Together, these findings are consistent with the hypothesis that preparatory aspects of feeding behaviors are preferentially associated with changes in DA metabolism and/or release in the NAc (Blackburn et al, 1989). Preparatory behaviors, including searching for food and water, are flexible patterns of responding that lead to and facilitate consummatory behaviors. These latter behaviors have rigidly defined patterns of motor activity, can be described objectively, and occur after an animal has made contact with a reward stimulus such as food (Konorski, 1967; Phillips et al, 1993).

Following withdrawal from an ED schedule of D-amph, DA efflux was significantly attenuated only during the initial 5 -min period of the preparatory phase of the sucrose test when compared to the pred-amph treatment (Figure 2c). In contrast, the increase observed during the first $5 \mathrm{~min}$ of the consummatory phase $(+32 \%)$ of the sucrose test was comparable to values obtained before treatment with D-amph (+27\%). These neurochemical data may explain our previous finding that withdrawal from an ED schedule of D-amph decreased selectively the preparatory components of sexual behavior in sexually experienced male rats (Barr et al, 1999). Previous in vivo microdialysis studies by our group have reported an immediate increase in DA levels in the NAc of sexually experienced male rats in the presence of an inaccessible receptive female (Fiorino et al, 1997; Fiorino and Phillips, 1999), which suggests that activity within the mesocorticolimbic DA pathway may be especially 
important for the initiation of motivated behavior. Furthermore, it is this rapid rise in DA efflux in the NAc that may be especially sensitive to the disruptive effects of withdrawal from extended exposure to psychostimulant drugs.

In addition to effects on sexual behavior, withdrawal from an ED schedule of D-amph has significant effects on other sensitive tests of motivated behavior. These include a significant reduction in break points under a progressive ratio schedule for a sucrose reward (Barr and Phillips, 1999; Orsini et al, 2001). It must be noted however that Russig et al (2003), in a study that restricted the ED schedule of $\mathrm{D}$-amph to a maximum dose of $5 \mathrm{mg} / \mathrm{kg}$, failed to replicate this effect. Amphetamine withdrawal also causes an elevation in brain reward thresholds in rats as measured by intracranial self-stimulation (Geyer and Markou, 1995). Furthermore, rats treated with an ED regimen of D-amph failed to display successive positive contrast when shifted from 4 to $32 \%$ sucrose (Vacca and Phillips, 2005), a finding that parallels the enhancement of successive negative contrast observed in rats withdrawn from the same drug treatment (Barr and Phillips, 2002). Genn et al (2004) have recently shown that DA efflux in the NAc was significantly attenuated when rats unexpectedly experience a reward of a lesser value in a negative contrast paradigm. Together, these data indicate that short-term abstinence from psychostimulants has a significant effect on the motivation to respond for natural rewards. This in turn may represent a preclinical analogue of the anhedonic state observed in humans during short-term abstinence from repeated high doses of D-amph or cocaine.

In a recent review of the role of the brain reward system in depression, Naranjo et al (2001) expanding on an earlier proposal by Blackburn et al (1992), argue that the mesolimbic DA pathway, rather than mediating hedonic responses per se, serves instead to induce approach (or preparatory) behaviors essential for consumption, as well as positive reinforcement and learning. Accordingly, DA neurons may be active during the encoding and retrieval of the positive value of an object, behavioral act, or internal physical state, essential for behavioral adaptation in a complex environment. According to this perspective, anhedonia may be redefined as state in which motivation is severely compromised. On the basis of the present data, we can also add that anhedonia induced by withdrawal from psychostimulant drugs may be a direct consequence of a reduced release of DA evoked by cues associated with natural reward stimuli or drug rewards. In conclusion, this study supports the continued use of preclinical psychostimulant withdrawal models of depression and emphasizes the need for further research on the role of brain DA systems in mood disorders.

\section{ACKNOWLEDGEMENTS}

We gratefully acknowledge Kitty So for her invaluable assistance during the microdialysis experiments. This research was funded by a grant from the Canadian Institutes of Health Research to AGP. GV was supported by a fellowship from the University of British Columbia, Faculty of Graduate Studies.

\section{REFERENCES}

Ahn S, Phillips AG (1999). Dopaminergic correlates of sensoryspecific satiety in the medial prefrontal cortex and nucleus accumbens of the rat. J Neurosci 19: RC29.

Barr AM, Fiorino DF, Phillips AG (1999). Effects of withdrawal from an escalating dose schedule of D-amphetamine on sexual behavior in the male rat. Pharm Biochem Behav 64: 597-604.

Barr AM, Markou A, Phillips AG (2002). A 'crash' course on psychostimulant withdrawal as a model of depression. Trends Pharm Sci 23: 475-482.

Barr AM, Phillips AG (1999). Withdrawal following repeated exposure to $\mathrm{D}$-amphetamine decreases responding for a sucrose solution as measured by a progressive ratio schedule of reinforcement. Psychopharmacology 141: 99-106.

Barr AM, Phillips AG (2002). Increased successive negative contrast in rats withdrawn from an escalating dose schedule of D-amphetamine. Pharmacol Biochem Behav 71: 293-299.

Blackburn JR, Pfaus JG, Phillips AG (1992). Dopamine functions in appetitive and defensive behaviours. Prog Neurobiol 39: 247-279.

Blackburn JR, Phillips AG, Jakubovic A, Fibiger HC (1989). Dopamine and preparatory behavior: II. A neurochemical analysis. Behav Neurosci 103: 15-23.

Di Chiara G, Loddo P, Tanda G (1999). Reciprocal changes in prefrontal and limbic dopamine responsiveness to aversive and rewarding stimuli after chronic mild stress; implications for the psychobiology of depression. Biol Psychiatry 46: 1624-1633.

Everitt BJ, Parkinson JA, Olmstead MC, Arroyo M, Robledo P, Robbins TW (1999). Associative processes in addiction and reward. The role of amygdala-ventral striatal subsystems. Ann NY Acad Sci 877: 412-438.

Fiorino DF, Coury A, Phillips AG (1997). Dynamic changes in nucleus accumbens dopamine efflux during the Coolidge effect in male rats. J Neurosci 17: 4849-4855.

Fiorino DF, Phillips AG (1999). Facilitation of sexual behavior in male rats following $d$-amphetamine-induced behavioral sensitization. Psychopharmacology 142: 200-208.

Gardner EL (2000). What we have learned about addiction from animal models of drug-self-administration. Am J Addict 9: 285-313.

Gawin F, Kleber H (1986). Pharmacologic treatments of cocaine abuse. Psychiatr Clin N Am 9: 573-583.

Genn RF, Ahn S, Phillips AG (2004). Attenuated dopamine efflux in the rat nucleus accumbens during successive negative contrast. Behav Neurosci 118: 869-873.

Geyer MA, Markou A (1995). Animal models of psychiatric disorders. In: Bloom FE, Kupfer DJ (eds). Psychopharmacology. Raven Press: New York. pp 797-798.

Kampman K, Volpicelli JR, McGinnis DE, Alterman AI, Weinrieb R, D'Angelo L et al (1998). Reliability and validity of the cocaine selective severity assessment. Addicit Behav 23: 449-461.

Kokkinidis L, Zacharko RM, Predy PA (1980). Post-amphetamine depression of self-stimulation responding from the substantia nigra: reversal by tricyclic antidepressants. Pharm Biochem Behav 13: 379-383.

Konorski J (1967). The Integrative Activity of the Brain. University of Chicago Press: Chicago.

Koob GF, Sanna PP, Bloom FE (1998). Neuroscience of addiction. Neuron 21: 467-476.

Lin D, Koob GF, Markou A (1999). Differential effects of withdrawal from chronic amphetamine or fluoxetine administration on brain stimulation reward in the rat: interactions between the two drugs. Psychopharmacology 145: 283-294.

Markou A, Koob GF (1991). Postcocaine anhedonia: an animal model of cocaine withdrawal. Neuropsychopharmacology 4: 17-26. 
Mogenson GJ, Phillips AG (1978). Brain-stimulation reward after twenty-five years. Can J Psychol 32: 54-57.

Naranjo CA, Tremblay LK, Busto UE (2001). The role of the brain reward system in depression. Prog Neuropsychopharmacol Biol Psychiatry 25: 781-823.

Orsini C, Koob GF, Pulvirenti L (2001). Dopamine partial agonist reverses amphetamine withdrawal in rats. Neuropsychopharmacology 25: 789-792.

Paxinos G, Watson C (1997). The Rat Brain in Stereotaxic Coordinates. Academic Press: New York.

Phillips AG, Atkinson LJ, Blackburn JR, Blaha CD (1993). Increased extracellular dopamine in the nucleus accumbens of the rat elicited by a conditional stimulus for food: an electrochemical study. Can J Physiol Pharmacol 71: 387-393.

Phillips AG, Fibiger HC (1978). The role of dopamine in maintaining intracranial self-stimulation in the ventral tegmentum, nucleus accumbens, and medial prefrontal cortex. Can $J$ Psychol 32: 58-66.

Russig H, Pezze MA, Nanz-Bahr NI, Pryce CR, Feldon J, Murphy CA (2003). Amphetamine withdrawal does not produce a depressive-like state in rats as measured by three behavioural tests. Behav Pharmacol 14: 1-18.

Ryan LJ, Linder JC, Martone ME, Groves PM (1990). Histological and ultrastructural evidence that D-amphetamine causes de- generation in the neostriatum and frontal cortex of rats. Brain Res 518: 67-77.

Srisurapanont M, Jarusuraisin N, Jittiwutikan J (1999). Amphetamine withdrawal: II. A placebo-controlled, randomised, doubleblind study of amineptine treatment. Aust NZ J Psychiatry 33: 94-98.

Vacca G, Phillips AG (2005). Inhibition of successive positive contrast in rats withdrawn from an escalating dose schedule of D-amphetamine. Int J Comp Psychol 18: 298-306.

Weddington WW, Brown BS, Haertzen CA, Cone EJ, Dax EM, Herning RI et al. (1990). Changes in mood, craving, and sleep during short-term abstinence reported by male cocaine addicts. Arch Gen Psychiatry 47: 861-868.

Weiss F, Markou A, Lorang MT, Koob GF (1992). Basal extracellular dopamine levels in the nucleus accumbens are decreased during cocaine withdrawal after unlimited-access selfadministration. Brain Res 593: 314-318.

Wilson C, Nomikos GG, Collu M, Fibiger HC (1995). Dopaminergic correlates of motivated behavior: importance of drive. J Neurosci 15: $5169-5178$.

Wise RA (1978). Catecholamine theories of reward: a critical review. Brain Res 152: 215-247.

Wise RA (1998). Drug-activation of brain reward pathways. Drug Alcohol Depend 51: 13-22. 